\title{
Fauna terrestre de Isla Riesco, Magallanes: una revisión bibliográfica
}

\author{
Terrestrial fauna of Riesco Island, Magallanes: \\ a bibliographic review
}

Javier A. Simonetti1 ${ }^{1,2}$ \& Gregor J. Stipicic ${ }^{2,3}$

\section{Resumen}

Se realizó una revisión de la literatura sobre la fauna terrestre reciente de Isla Riesco, Magallanes. De 60 publicaciones, que cubren los últimos 140 años, extrajimos la información sobre los taxones, temas y lugares estudiados.

\section{Palabras clave:}

arácnidos, insectos, peces, anfibios, mamíferos, aves.

\section{Abstract}

We performed a review of the literature on recent terrestrial fauna of Riesco Island, Magallanes. Information regarding taxa studied, topics covered, and locality of study was retrieved from 60 publications spanning the last 140 years.

\section{Key words:}

arachnids, insects, fish, amphibians, mammals, birds.

\section{INTRODUCCIÓN}

Isla Riesco $\left(53^{\circ} 00^{\prime} \mathrm{S} 72^{\circ} 30^{\prime} \mathrm{O}\right)$, con una superficie de $5.005 \mathrm{~km}^{2}$, es la cuarta isla más grande de Chile a continuación de la Isla Grande de Chiloé, Isla Grande de Tierra del Fuego e Isla Wellington. La isla ha sido fuente de inspiración para estudios de los territorios magallánicos, y aloja el sitio arqueológico de Ponsonby, clave para entender el poblamiento regional y las transiciones culturales desde cazadores terrestres a nómades marinos (Solari et al. 2002; Lefèvre et al. 2003; Martinic, 2011). Ella ha sido, además, foco de estudios de geología, glaciología y flora (e.g.,
Casassa et al. 2002; Leppe et al. 2012; Betka et al . 2016). Actualmente, Isla Riesco es foco de estudio tanto porque las actuales operaciones mineras son consideradas un caso de injusticia ambiental (Bustos et al. 2017), como por el significado social y ambiental de la ampliación y transformación de la Reserva Nacional Alacalufes en el Parque Nacional Kawésqar (Zorondo-Rodríguez et al. 2019).

Isla Riesco también ha sido objeto de estudios tanto de fauna actual como fósil (e.g., Markham, 1970; Hünicken et al. 1980), inspirando incluso poemas, como "Tuco tuco de la Isla Riesco" (Silva, 2002). Si bien existen publicaciones de difusión y generales sobre la biota de la región de Magallanes, incluyendo aquella de Isla Riesco (e.g. Martinic, 1957; Markham, 1971a; Venegas \& Sielfeld, 1998), no existe a la fecha una síntesis de la misma. Con objeto de contribuir a sistematizar la información disponible sobre la fauna terrestre de Isla Riesco, entregamos una reseña de las publicaciones disponibles sobre la materia, presentando los grupos taxonómicos y temas abordados a la fecha. Esperamos que esta síntesis contribuya a orientar los futuros trabajos sobre la biodiversidad de Isla Riesco.

\footnotetext{
1 Departamento de Ciencias Ecológicas, Facultad de Ciencias, Universidad de Chile. $\measuredangle$ jsimonet@uchile.cl

2 Asociación Kauyeken, Isla Riesco \& Santiago, Chile.

3 Estancia Anita Beatriz, km 35 Ruta Y-560, Isla Riesco, Magallanes, Chile.
} 


\section{MÉTODOS}

Realizamos una búsqueda de publicaciones sobre la fauna contemporánea de la isla en diferentes buscadores, incluyendo Ebsco, Jstor, Google Académico, Scopus y Web of Science. Para dichas búsquedas empleamos los términos "Isla Riesco" y "Riesco Island". Adicionalmente revisamos las referencias de las publicaciones recopiladas para completar la búsqueda de trabajos atingentes a la fauna terrestre de la isla. Excluimos la literatura paleontológica, ya reunida en Pérez (2018) y Pérez y Pérez (2018), al igual que aquella encontrada en contextos arqueológicos, como la presencia de Lama guanicoe en Isla Riesco (San Román et al. 2002; Lepetz et al. 2003), la que será reseñada en forma independiente.

De cada publicación presentamos una breve reseña de su contenido, ordenadas por taxón en orden alfabético. Si el o los taxones tratados en un trabajo son referidos por trabajos posteriores o en curso, éstos se reseñan. Hemos actualizado la nomenclatura original empleada por los autores en caso de haber modificaciones taxonómicas desde la fecha de publicación del trabajo.

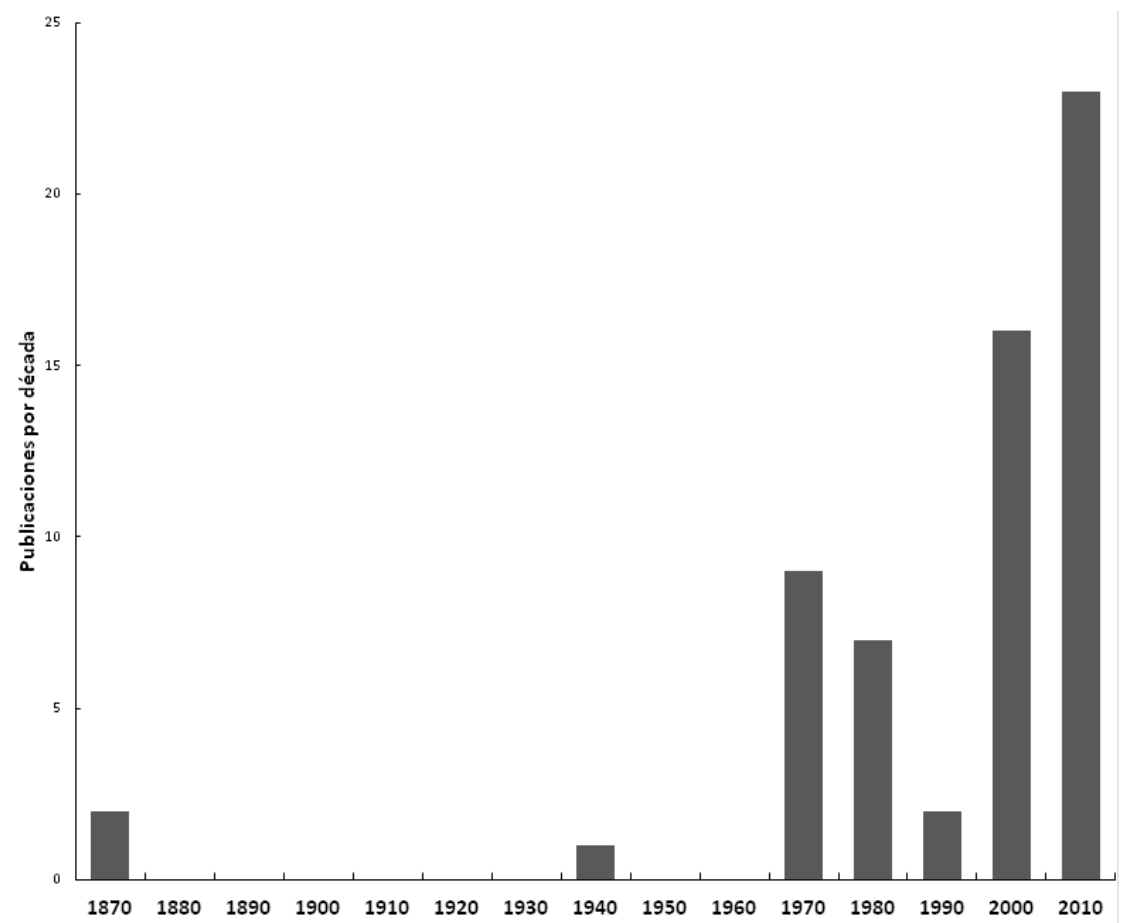

Fig. 1. Número de estudios (publicados y en curso) sobre fauna terrestre en Isla Riesco, Magallanes.

\section{RESULTADOS}

Recopilamos 60 publicaciones, entre artículos científicos, libros e informes, las que comprenden un período de 140 años, desde 1878 con las notas del naturalista Enrique Ibar (Pisano, 1977). $\mathrm{Si}$ bien no pretendemos que esta revisión haya cubierto todos y cada uno de los documentos que hagan referencia a la fauna de Isla Riesco, destaca que $65 \%$ de las publicaciones recopiladas se han producido en las últimas dos décadas (Fig. 1). Los mamíferos concentran la mayor parte de los estudios publicados y en curso (57\%). Insectos (18\%) y aves (15\%) son estudiados en menor proporción, en tanto arácnidos, peces y anfibios son escasamente reseñados (Tabla 1). La mayor parte de los trabajos (37\%) aborda aspectos referidos a la distribución, sean nuevos hallazgos para la isla o catastros de la fauna de un sector particular de la isla, o bien cubre aspectos de la taxonomía o sistemática (27\%), principalmente de insectos y mamíferos (Tabla 1). La mayor parte de los trabajos no presenta detalles del sector de Isla Riesco donde se colectaron los ejemplares estudiados o bien son poco precisos (e.g. sector este de la isla). La Estancia Anita Beatriz es 
Tabla 1. Número de estudios (publicados y en curso) sobre diferentes taxones y temas abordados en la fauna terrestre de Isla Riesco, Magallanes. El número de temas es mayor al de estudios pues un trabajo puede abordar más de un tema.

\begin{tabular}{|c|c|c|c|c|c|c|c|}
\hline & Arácnidos & Insectos & Peces & Anfibios & Mamíferos & Aves & $\begin{array}{l}\text { Total } \\
\text { temas }\end{array}$ \\
\hline $\begin{array}{l}\text { Taxonomía } \\
\text { Sistemática }\end{array}$ & 4 & 8 & & & 6 & 1 & 19 \\
\hline Distribución & 1 & 1 & 1 & 2 & 10 & 8 & 23 \\
\hline $\begin{array}{c}\text { Historia natural \& } \\
\text { Ecología }\end{array}$ & & 4 & & & 7 & 1 & 12 \\
\hline Enfermedad & & & & & 3 & & 3 \\
\hline $\begin{array}{l}\text { Conflictos con } \\
\text { fauna }\end{array}$ & & & & & 7 & & 7 \\
\hline Gestión & & & & & 2 & & 2 \\
\hline $\begin{array}{l}\text { Total Publicaciones } \\
\text { y estudios en curso }\end{array}$ & 3 & 11 & 1 & 2 & 34 & 9 & $60 / 63$ \\
\hline
\end{tabular}

el sector que ha recibido mayor atención tanto en cantidad de estudios como diversidad de taxones estudiados (Fig. 2). Ponsonby es el segundo sitio con mayor cantidad de estudios, incluyendo arácnidos y mamíferos (Fig. 2). Todos estos trabajos se presentan a continuación por taxón.

\section{ARÁCNIDOS}

Grismado y Platnick (2008) redescriben Platnickia bergi (Simon) sobre la base, entre otros, de un ejemplar colectado en Ponsonby. Proveen una clave para la determinación de las diferentes especies del género Platnickia Jocqué, 1991.

Ramírez (2003), en su revisión de la subfamilia Amaurobioidinae, propone a Negayan paduana (Karsch) y Sanogasta backhauseni (Simon) como nuevas combinaciones empleando, entre otros, ejemplares provenientes de Ponsonby y Vaquería. Indica también que $N$. paduana construye sus refugios bajo piedras o cortezas de troncos caídos, en tanto la historia natural de $S$. backhauseni es desconocida.

Taucare-Ríos (2014) presenta el primer registro de Mecysmauchenius segmentatus
Simon, 1884 en la isla, basado en un ejemplar colectado en Estancia Anita Beatriz, ampliando su distribución dentro de la región de Magallanes, proponiendo que sería frecuente en el bosque subpolar magallánico, incluyendo bosques sometidos a pastoreo ovino.

\section{INSECTOS}

Alvarado (2013) incluye ejemplares de Isla Riesco en la descripción de una nueva especie innominada del género Alophophion Cushman, 1947 (Alophophion nueva especie 19) entre las 38 especies nuevas que describe. Posteriormente Alvarado (2014) la nomina Alophophion yagane. En ambas publicaciones provee una clave para la determinación de los diferentes grupos de especies y especies del género Alophophion, pero no señala la localidad desde donde se obtuvieron los ejemplares.

Cekalovic (1981) describe la larva y pupa de Ceroglossus suturalis (Fabr., 1775) basado en ejemplares de Mina Elena, nueva localidad de colecta de la especie, y entrega datos generales sobre el hábitat donde ocurre C. suturalis. Los 


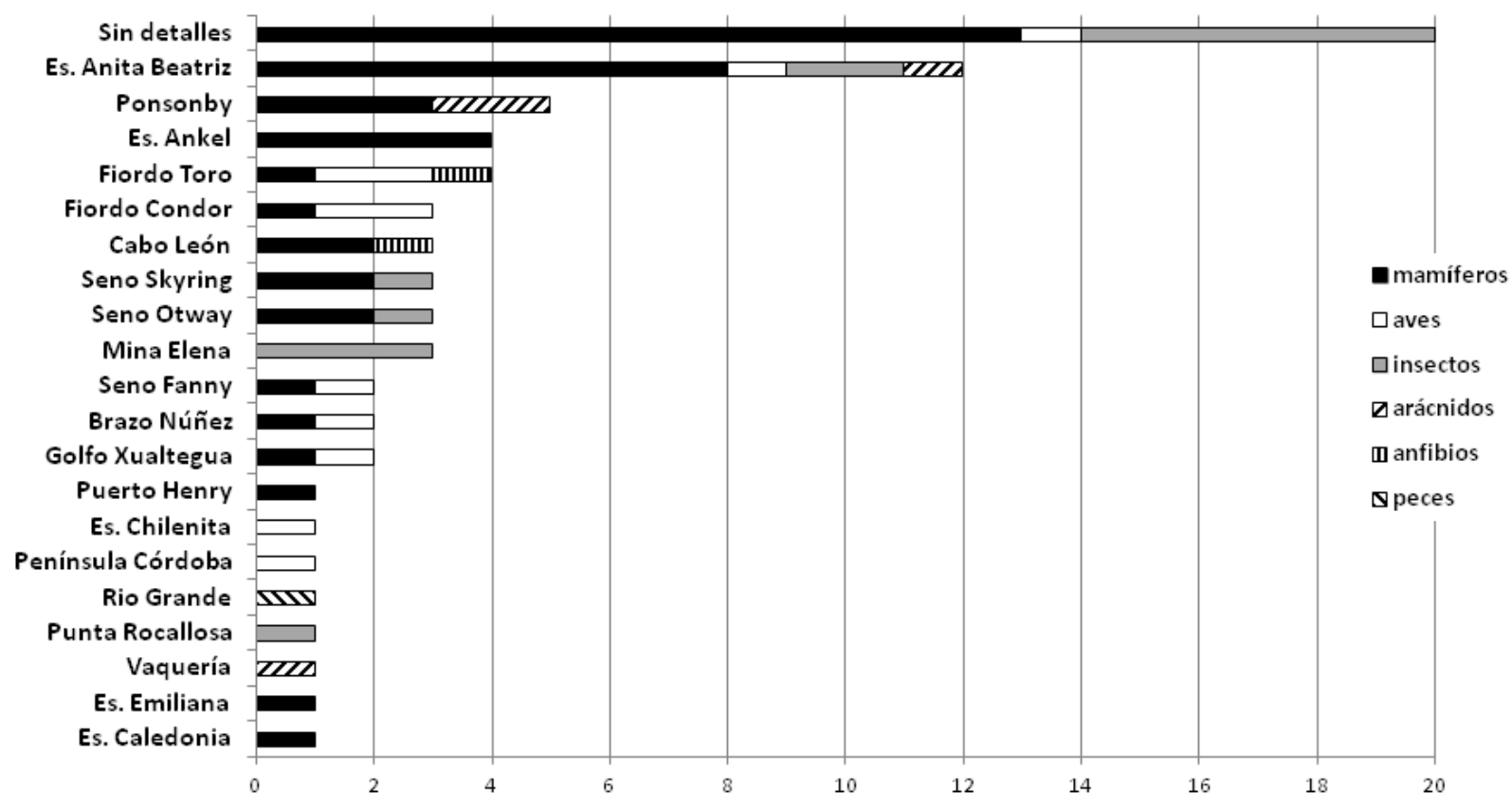

Fig. 2. Lugares de registros, colectas o estudios de campo (publicados y en curso) sobre diferentes taxones de fauna terrestre en Isla Riesco, Magallanes. Es: estancia.

ejemplares son asignados como nepionotipo de la especie en Cekalovic y Artigas (1981).

Cekalovic (1982) describe la larva y pupa de Erichius femoralis (Guérin-Méneville, 1839) (Pycnosiphorus femoralis en el original) basado, entre otros, en ejemplares colectados en el sector de Mina Elena. Además, entrega datos generales sobre el hábitat donde ocurre E. femoralis.

Salgado (2005, 2006) estudia ejemplares de Falkocholeva cribellata (Fairmaire \& Germain, 1859) en su revisión de la tribu Eunemadina y subfamilias Camiarinae y Cholevinae. Describe por vez primera los $7^{\circ}$ y $8^{\circ}$ esternitos de los segmentos abdominales femeninos para facilitar la diferenciación de otras especies. Asimismo, entrega una clave para los géneros latinoamericanos de Eunemadina, pero no señala la localidad desde donde se obtuvieron los ejemplares.

Simonetti et al. (2016) comunican la presencia de ejemplares asilvestrados de Bombus terrestris (Linnaeus, 1758) en Estancia Anita Beatriz y describen su hábitat y abundancia comparada con Bombus dahlbomii GuérinMéneville, 1835 catalogándola como una amenaza a la fauna de polinizadores nativos.

Snelling y Hunt (1975) revisan ejemplares de Monomorium denticulatum (Mayr, 1887) (Antichthonidris denticulatus en el original) provenientes del sector de Mina Elena para su revisión de las hormigas de Chile.

Zúñiga-Reinoso (2011) revisa los coccinélidos magallánicos y colecta ejemplares de Eriopis magellanica (Philippi \& Philippi, 1864) en seno Skyring y seno Otway, y de Orynipus kuscheli Hofmann, 1972 en punta Rocallosa. Además, entrega datos sobre sus hábitats y hábitos.

Zúñiga-Reinoso (2013) en su revisión de los cerambícidos de Magallanes indica la presencia de Lautarus concinnus (Philippi \& Philippi, 1859) en Isla Riesco, indicando que es una especie rara con pocos registros en la región, sin precisar el lugar de colecta.

\section{PECES}

Correa y Gross (2008) documentan la invasión de Oncorhynchus tshawytscha en el sur de Sudamérica, incluyendo el río Grande en Isla Riesco durante el año 2004, realizando inferencias tanto sobre las condiciones ambientales que facilitarían su expansión en la región como sobre las consecuencias socio-ambientales de la misma. 


\section{ANFIBIOS}

Aguayo et al. (2007) citan la presencia de Chaltenobrachus aff. grandisonae (Atelognathus en el original) en fiordo Toro. Sin embargo, el mismo registro aparece como Batrachyla sp. en Frangópulos (2007). Esto corresponde a una confusión con información bibliográfica sobre las potenciales especies de anfibios señaladas como posibles de encontrarse en la región (J. C. Ortíz, comunicación personal, julio 2019). En efecto, este registro es nuevamente referido como $C$. aff. grandisonae en la revisión de los anfibios de las turberas australes (Ortiz, 2015). Ejemplares de Batrachyla cf. antartandica han sido observados en cabo León (C. Magallanes, G. Simonetti-Grez, G. J. Stipicic \& S. Fortes, datos no publicados).

\section{MAMÍFEROS}

Belmar-Lucero et al. (2009) analizan el cariotipo y secuencian la región hipervariable I del mtDNA de Oligoryzomys de la Patagonia sur, incluyendo Isla Riesco. El cariotipo de los ejemplares al sur de los $51^{\circ} \mathrm{S}$ sería consistente con $O$. longicaudatus y no presentarían diferencias filogenéticas con aquellos de la región norte de la distribución. Con ello, amplían la distribución de esta especie hasta al menos $55^{\circ} \mathrm{S}$. Además, no detectan individuos seropositivos al virus Hanta entre los estudiados en Isla Riesco. Los mismos ejemplares estudiados por Belmar-Lucero et al. (2009) son luego analizados en un estudio sobre la sistemática de Oligoryzomys así como el efecto de las glaciaciones en su diversidad genética, proponiendo que las poblaciones al sur de los $45^{\circ}$ S serían O. l. magellanicus (Palma et al. 2012, Palma \& Rodríguez-Serrano, 2018).

Crespín (2018) analizó el efecto de la densidad de presas nativas (roedores y artrópodos) y exóticas (Lepus europaeus Pallas, 1778) sobre la tasa de depredación de ganado ovino por zorros (Lycalopex spp) en la Estancia Anita Beatriz y sugiere formas de gestión de presas nativas para reducir ataques a ganado ovino.

Durán et al. (1985) evaluaron la abundancia de L. griseus (Gray) (Canis en el original) en la costa este de Isla Riesco, incluyendo desde el seno Otway al Skyring, estimando densidades de 2,6 a 6,6 individuos por cada 100 ha en sectores costeros de la parte este de la isla. Proponen que el desmonte de vegetación para generar más hábitat para el ganado favorecería a estos zorros, indicando que la cacería ilegal sería una amenaza a la población.

Durán y Favi (1989) evalúan la existencia de rabia en L. griseus (Pseudalopex en el original) en la Región de Magallanes y Antártica Chilena, sin encontrar ejemplares con rabia en ejemplares de Isla Riesco, colectados en la misma zona estudiada por Durán et al. (1985). Al mismo tiempo analizan la prefactibilidad de la vacunación oral extensiva de zorros, quienes son considerados como los propagadores y víctimas principales de la rabia.

Graells et al. (2015) incluyen a Isla Riesco como un área que pudiese ser invadida por Castor canadensis Kuhl, 1820, considerando la capacidad de dispersión de individuos establecidos en Río Verde. Prospecciones realizadas en la isla hasta 2017 no han detectado señales de su presencia (Molina et al. 2018)

Markham (1970) realiza un reconocimiento de la fauna del área de los fiordos Toro y Cóndor, registrando la presencia de Lycalopex culpaeus culpaeus (Molina, 1782) (Dusicyon culpaeus en el original), Abrothrix cf. longipilis (Waterhouse, 1837) (Akodon cf. longipilis en el original), Loxodontomys micropus (Waterhouse, 1837) (Phyllotis micropus en el original) y Oligoryzomys longicaudatus philippii (Bennett, 1832) (Oryzomys longicaudatus en el original). La presencia de O.l. philippii expande su distribución en $3^{\circ}$ hacia el sur.

Markham (1971b), basado en el ejemplar colectado en Puerto Henry, propone que la subespecie de Lycalopex culpaeus de la isla correspondería a L. culpaeus culpaeus y sugiere que en Magallanes esta subespecie se encontraría probablemente restringida a los bosques siempreverdes de los canales en el oeste de la provincia. De igual forma, Guzmán et al. (2009) en base a dos ejemplares de Isla Riesco (localidad de colecta no indicada), entre otros, analizan la variación geográfica de esta especie de zorro, agrupando a todos los ejemplares del centrosur y Patagonia e islas australes en la subespecie culpaeus.

Ojeda y Pérez (2009) analizan el conocimiento de la presencia de Puma concolor 
(Linneo, 1771) y de la actividad "leonera" en Magallanes como parte del conflicto carnívorosganadería, incluyendo Isla Riesco. La magnitud de la cacería ilegal de $P$. concolor y Lycalopex spp. en Magallanes incluyendo Isla Riesco se encuentra en estudio (D. Peñaranda \& J. A. Simonetti, en curso) ${ }^{1}$ y la efectividad de la cacería ilegal para reducir este conflicto en la comuna de Río Verde, incluyendo Isla Riesco, ha sido analizada por Moreira-Arce et al. (2018). Por su parte, Díaz (2018) y Díaz et al. (2019) evaluaron el grado de aceptación social para diferentes técnicas de manejo de carnívoros nativos (L. culpaeus, L. griseus y P. concolor) y exóticos (Canis familiaris Linneo, 1758) y en diferentes escenarios de riesgo para el ganado ovino $y$ población humana. Moreira-Arce et al. (en curso) se encuentran analizando los factores a escala predial y de paisaje que inciden en los riesgos de depredación ovina por parte de carnívoros nativos. Estos trabajos se realizan en las Estancias Adela, Anita Beatriz, Ankel, Caledonia y Emiliana.

En la misma línea, Saavedra (2018) determinó que el uso de repelentes olfativos aumenta la densidad de alimento abandonado por L. culpaeus y L. griseus en Estancia Anita Beatriz, y aumenta sus conductas de vigilancia, sugiriendo que el uso de repelentes podría reforzar el efecto protector de los perros pastores o perros de guarda. Actualmente Ugarte (en curso) analiza el efecto de repelentes olfativos basados en olor corporal completo de perros protectores de ganado ( $C$. familiaris) contra Lycalopex a escala de paisaje, incluyendo Estancia Anita Beatriz (C. Ugarte, datos no publicados).

Igualmente, A. P. Yusti (en curso) analiza si los perros protectores de ganado (C. familiaris) tienen efectos indirectos sobre la depredación en ovejas domésticas (Ovis aries), haciéndolas más alertas frente a depredadores nativos, ofreciendo una protección adicional a la exclusión de depredadores, contribuyendo a la conservación de los carnívoros silvestres al reducir el uso de control letal (Yusti, com. per.). También asociado al uso de perros protectores de ganado (C. familiaris), A.A. Arzabe está investigando los posibles efectos

1 Peñaranda, D. A., \& Simonetti, J. A. (2018). Hacia la resolución de los conflictos entre ganadería y carnívoros silvestres: evidencia sobre la efectividad de alternativas no letales. Simposio "Conservación y manejo de carnívoros subletales de su uso sobre Lycalopex, evaluando estrés, capacidad del sistema inmune, prevalencias parasitarias y adecuación biológica de Lycalopex (A. A. Arzabe, com. per.). Por último, R. Contreras (en curso) ha analizado si Lycalopex efectivamente reduce su consumo de ganado ovino (O. aries) en presencia de perros protectores. Todos estos trabajos se desarrollan actualmente en las Estancias Anita Beatriz y Ankel.

Osgood (1943) describe la subespecie Ctenomys magallenicus dickii con dos ejemplares colectados en la Estancia Ponsonby en 1940, la cual describe como confinada al sector este de la isla donde indica que son raros no obstante existir numerosas cuevas. Carlos Hoffmann comunica observar un ejemplar muerto en la misma zona en 1928 (Martinic, 2005). La presencia de cuevas pero la escasez de ejemplares ya era observada en 1877 en las costas continentales de los senos Otway y Skyring (Ibar, 1878; véase además Latorre, 1879), donde C. magallenicus no se ha extinto como lo estaría la subespecie dickii en Isla Riesco. Así, mientras Miller et al. (1983) indican que C. $m$. dickii sería rara, sin entregar otros antecedentes, Gallardo (1979), en 1976, no encuentra rastro alguno de Ctenomys en la misma zona donde Osgood (1943) obtiene los ejemplares sobre los que describe C. m. dickii, declarándola extinta. Gallardo (1979) atribuye al pastoreo intenso y otros posibles factores su desaparición. La subespecie ha sido declarada Extinta de acuerdo a la Clasificación de Especies según su Estado de Conservación (MMA, 2013).

Osgood (1943) reporta ejemplares de Akodon xanthorhinus xanthorhinus (Waterhouse, 1837) capturados en el extremo este de la isla, señalando que donde se encuentran suelen ser abundantes.

Osgood (1943), basado en informes que considera confiables, señala que Pudu puda (Molina, 1782) ( $P$. pudu en el original) ha sido visto o capturado en el lado occidental de la isla, tan al sur como los $50^{\circ} \mathrm{S}$, indicando que ningún ejemplar habría sido preservado. Igualmente, Tamayo y Frassinetti (1980) mencionan P. puda en la isla,

andinos". V Congreso Latinoamericano y VIII Congreso Boliviano de Mastozoología. Cochabamba, Bolivia, 10-13 Julio. 
sin aportar antecedentes. Jiménez (2010), en una revisión de la información conocida para la especie, pone en duda esta afirmación. Muestreos en curso por nuestro equipo de trabajo en diferentes sectores de la isla no han registrado esta especie.

Povilitis y Hughes (2003) evalúan la abundancia y el uso del hábitat por Hippocamelus bisulcus (Molina, 1782) en el sector del río León, actualmente Parque Nacional Kawésqar, estimando una densidad de 0,3 individuos $/ \mathrm{km}^{2}$, señalando que todas las señas de huemules fueron registradas en bosques, áreas con arbustos densos y en laderas de inclinación moderada o alta. También proponen que perturbación humana directa o mediada por ganado explicaría esta aparente baja abundancia. Recientemente se ha registrado una abundancia mayor en la misma área (Asociación Kauyeken, datos no publicados) $)^{2}$. Astas de $H$. bisulcus han sido encontradas en 1928 (según las cartas del Sr. C. Hoffmann) en el área de la Estancia Ponsonby y astas antiguas en Estancia Ankel (Martinic, 2005; D. Moreira-Arce \& D. Peñaranda, com. per.).

Sielfeld para Aonken Consultores Ltda. (1982), en base a informantes locales, indica que en los sectores boscosos de la zona sur de la isla existirían huemules, donde sería frecuente observar piños de hasta cinco ejemplares. Un ejemplar juvenil colectado en 1980 en la zona sur se conserva en la colección del Instituto de la Patagonia, al igual que un ejemplar de Myocastor coypus, colectado en un lugar no determinado de la isla.

Por su parte, Silva et al. (2011) solicitan que todo proyecto a desarrollarse en hábitats con presencia histórica de H. bisulcus (Molina, 1782) sea sometido a una Evaluación de Impacto Ambiental, requiriendo que se incorporen medidas adecuadas para resguardar la totalidad de la población en el área de ejecución del actual proyecto minero en Isla Riesco. Posteriormente, Kusch et al. (2016) añaden que deberían desarrollarse buenas prácticas de manejo agroforestal en áreas con presencia de $H$. bisulcus, incluyendo Isla Riesco, y que debería evaluarse la variabilidad genética de esta especie, teniendo como prioridad el área de cabo Froward (península Brunswick) e Isla Riesco.

Rodríguez-Serrano (2011) analiza las consecuencias de las glaciaciones sobre Abrothrix

2 https://laprensaaustral.cl/cronica/rodrigo-perez-trashallazgos-de-esta-especie-en-isla-riesco-la-integracion-de- olivaceus (Waterhouse, 1837), proponiendo la presencia de múltiples refugios glaciales en el Bosque Valdiviano, la Estepa Patagónica, la Patagonia occidental y en islas Riesco y Tierra del Fuego, lo cual explicaría su heterogeneidad genética a través de la Patagonia. La población de Isla Riesco estaría estrechamente relacionada a las poblaciones de Tierra del Fuego, con baja diversidad haplotípica, aisladas por el canal Fitz Roy, lo que habría conllevado un fuerte cuello de botella. La subespecie presente en Isla Riesco sería A. o. xanthorhinus.

Sielfeld para Aonken Consultores Ltda. (1982) prospectó la fauna del golfo Xaultegua, brazo Núñez y estero Fanny. En base a capturas, fecas y restos detectan Abrothrix longipilis (Akodon en el original), Loxodontomys micropus (Phyllotis en el original) y Oligoryzomys longicaudatus (Oryzomys en el original), y proponen que se les encontraría preferentemente en sectores boscosos. La presencia de Myocastor coypus (Molina, 1782) es comunicada en base a un ejemplar colectado en un sitio no determinado de la isla, en depósito en la colección del Instituto de la Patagonia.

En las áreas prospectadas, registra Lycalopex culpaeus (Canis en el original) pero no así L. griseus. Asimismo, comunica la presencia de Lontra provocax (Thomas, 1908) (Lutra en el original), la que ocuparía la zona de bosques costeros siempreverdes y cuyo hábitat requeriría de protección, dentro de la entonces Reserva Nacional Alacalufes, actualmente Parque Nacional Kawésqar (ver también Sielfeld, 1997).

Sielfeld (1979) cuestiona la proposición de Mann (1978) respecto a la presencia de Microcavia australis (G. y D'O) en Isla Riesco por falta de registros fehacientes.

\section{AVES}

Couve y Vidal (2003) mencionan a Molothrus bonariensis (Gmelin, 1789) en Isla Riesco, sin embargo, Jaramillo et al. (2003) indican que llegaría solamente hasta Aysén, no obstante, existe un registro en Primera Angostura, más al sur de Isla Riesco (Matus \& Jaramillo, 2008).

Kusch y Marín (2010) indican la presencia

cabo-leon-al-futuro-parque-nacional-kawesqar-contribuiraa-garantizar-la-proteccion-del-habitat-del-huemul/ 
de Gallinago stricklandii (Gray, 1845) en estero Cóndor, en base a un ejemplar observado en el verano del 2010, resaltando la baja frecuencia con que la especie es registrada en la zona austral de Chile.

Markham (1970) prospecta las aves de los fiordos Toro y Cóndor, extremo sudoeste de Isla Riesco, reseñando la presencia de 27 especies de aves y adelantando la descripción de una nueva subespecie de tordo Curaeus curaeus recurvirostris subsp. nov. Describe además el contenido estomacal de varias especies, entregando otros datos de su historia natural (ubicación, abundancia relativa, parásitos).

Markham (1971c) propone formalmente la nueva subespecie de tordo, Curaeus curaeus recurvirostris Markham 1971, basado en su mayor tamaño y forma diferente del pico respecto de la subespecie nominal, basado en ejemplares provenientes del fiordo Toro. Imberti (2005), sin embargo, no registró ejemplares de esta subespecie en varias visitas (no detalladas) a la isla, desde el río Grande al este, no obstante observar numerosos ejemplares de C. curaeus (Molina, 1782).

Sielfeld para Aonken Consultores Ltda. (1982) evalúa la ornitofauna en golfo Xaultegua, brazo Núñez y seno Fanny, además de aves marinas, mencionan a Chloephaga hybrida (Molina, 1782), Tachyeres pteneres (Forster, 1844) y T. patachonicus (King, 1831), Lophonetta specularioides (King, 1828), Caracara plancus (Miller, 1777) (Polyborus plancus en el original), Megaceryle torquata (Linnaeus, 1766) (Ceryle torquata en el original), Cinclodes patagonicus (Gmelin, 1789) y C. curaeus en litoral. En matorral y bosque mencionan Sephanoides sephaniodes (Lesson \& Garnot, 1827) (S. galeritus en el original), Aphrastura spinicauda (Gmelin, 1789), Scytalopus magellanicus (Gmelin, 1789), Xolmis pyrope (Kittlitz, 1830) (Pyrope pyrope en el original), Pygarrhichas albogularis (King, 1831), C. curaeus, Spinus barbatus (Molina, 1782) (Carduelis barbatus en el original), Enicognathus ferrugineus (Muller, 1776) y Milvago chimango (Vieillot, 1816). De Bubulcus ibis (Linnaeus, 1758 ) indica que es un ave visitante que no anida en la isla.

Simonetti y Stipicic (2016) registran por primera vez Phytotoma rara (Molina, 1782) en
Estancia Anita Beatriz, sugiriendo un progresivo avance de la especie hacia el sur en virtud a que los registros más meridionales son más recientes.

Stipicic et al. (2017) registran por primera vez una pareja de Chloephaga rubidiceps (Sclater, 1861) frente a la Estancia Chilenita, sugiriendo que el canquén colorado podría nidificar en la isla pues se registró en época reproductiva.

Wheelwright (1978) comunica la presencia de Egretta thula (Molina, 1782) frente a península Córdoba, ampliando en más de $1.000 \mathrm{~km}$ el rango de distribución conocida, aun cuando pudiese ser solamente un visitante casual.

\section{COMENTARIOS FINALES}

La fauna terrestre de Isla Riesco ha comenzado a recibir creciente atención, como lo ha hecho en el pasado su fauna en contextos zooarqueológicos (e.g., Legoupil, 2003). Sin embargo, la fauna de la cuarta isla más grande del país comparativamente ha sido objeto de menos estudios que otras áreas de Chile. Ejemplo de ello es la cantidad de estudios realizados en el sector de San Carlos de Apoquindo, en la precordillera de Santiago, con una superficie equivalente solo al $0,16 \%$ de Isla Riesco. Sobre la fauna de esta área se publicaron 92 trabajos solamente entre 1976 y 2000, incluyendo artrópodos, herpetozoos, mamíferos y aves tanto desde un análisis de la composición y estructura de sus ensambles así como de las interacciones y procesos en los que participan (Jaksic, 2001). La creación del nuevo Parque Nacional Kawésqar podría ser un estímulo y facilitar los estudios sobre la fauna, sea en su composición, estructura y funcionamiento de manera de tener información sólida para planificar adecuadamente la gestión de este territorio y su patrimonio biológico.

\section{LITERATURA CITADA}

Aguayo, A. (ed.) (2007). Informe Final "Diagnóstico de la Flora y Fauna de vertebrados y Aves marinas del AMCP Francisco Coloane". Punta Arenas, Chile: Ministerio de Bienes Nacionales.

Alvarado, M. (2013). Revision of the South American wasp genus Alophophion 
Cushman, 1947 (Hymenoptera: Ichneumonidae: Ophioninae). Master Thesis. Kansas, USA: Department of Ecology and Evolutionary Biology, University of Kansas.

Alvarado, M. (2014). Revision of the South American wasp genus Alophophion Cushman, 1947 (Hymenoptera: Ichneumonidae: Ophioninae). Revista Peruana de Biología, 21, 003-060.

Aonken Consultores Ltda. (1982). Catastro y evaluación de recursos de las Reservas Forestales Alacalufes e Isla Riesco. Punta Arenas, Chile: Corporación Nacional Forestal.

Belmar-Lucero, S., Godoy, P., Ferres, M., Vial, P., \& Palma, R. E. (2009). Range expansion of Oligoryzomys longicaudatus (Rodentia, Sigmodontinae) in Patagonian Chile, and first record of Hantavirus in the region. Revista Chilena de Historia Natural, 82, 265-275.

Betka, P., Klepeis, K., \& Mosher, S. (2016). Fault kinematics of the Magallanes-Fagnano fault system, southern Chile; an example of diffuse strain and sinistral transtension along a continental transform margin. Journal of Structural Geology, 85, 130-153.

Bustos, B., Folchi, M., \& Fragkou, M. (2017). Coal mining on pastureland in Southern Chile; challenging recognition and participation as guarantees for environmental justice. Geoforum, 84, 292-304.

Casassa, G., Smith, K., Rivera, A., Araos, J., Schnirch, M., \& Schneider, C. (2002). Inventory of glaciers in Isla Riesco, Patagonia, Chile, based on aerial photography and satellite imagery. Annals of Glaciology, 34, 373-378.

Cekalovic, T. (1981). Descripción de la larva y pupa de Ceroglossus suturalis (Fabr.), 1775 (Coleoptera, Carabidae). Boletín de la Sociedad de Biología de Concepción, 51, 67-73.

Cekalovic, T. (1982). Descripción de la larva y pupa de Pycnosiphorus femoralis (Guerin 1839) (Coleoptera, Lucanidae). Boletín de la Sociedad de Biología de Concepción, $53,33-40$.
Cekalovic, T., \& Artigas, J. (1981). Catálogo de los tipos depositados en las colecciones del Departamento de Zoología de la Universidad de Concepción, Chile. Boletín de la Sociedad de Biología de Concepción, 52, 203-224.

Correa, C., \& Gross, M.R. (2008). Chinook salmon invade southern South America. Biological Invasions, 10, 615-639.

Couve, E., \& Vidal, C. (2003) Aves de Patagonia, Tierra del Fuego y Península Antártica. Punta Arenas, Chile: Fantástico Sur.

Crespin, S. J. (2018). Completing the landsharing strategy: reaching human-wildlife coexistence through alternative resource management. Tesis Doctoral. Santiago, Chile: Facultad de Ciencias, Universidad de Chile.

Díaz, M. V. (2018). Aceptación social de diferentes técnicas de manejo de carnivoros a través de distintos escenarios de conflicto entre la fauna silvestre y humanos. Seminario de Título. Santiago, Chile: Facultad de Ciencias, Universidad de Chile.

Díaz, M. V., Simonetti, J. A., \& ZorondoRodríguez, F. (2019). Social acceptability of management actions for addressing different conflict scenarios between humans and wildlife in Patagonia. Human Dimensions of Wildlife, en prensa.

Durán, J. C., \& Favi, M. (1989). Rabia en zorro gris (Pseudalopex griseus) patagónico, Magallanes, Chile. Avances en Ciencias Veterinarias, 4, 146-152.

Durán, J. C., Cattan, P. E., \& Yáñez, J. L. (1985). The grey fox Canis griseus (Gray) in Chilean Patagonia (southern Chile). Biological Conservation, 34, 141-148.

Frangópulos, M. (2007). Diagnóstico de flora, fauna terrestre y aves marinas en el Área Marina Costera Protegida Francisco Coloane. Punta Arenas, Chile: Informe Final, Fundación Cequa.

Gallardo, M. (1979). Las especies chilenas de Ctenomys (Rodentia, Octodontidae). I. Estabilidad cariotípica. Archivos de Biología y Medicina Experimental, 12, 71-82.

Graells, G., Corcoran, D., \& Aravena, J. C. (2015). Prospección y datación de la fecha 
de colonización del área del Río Hollemberg, provincia de Última Esperanza, el punto más septentrional de presencia de Castor canadensis (Castoridae) en Sudamérica. Anales del Instituto de la Patagonia, 43, 61-67.

Grismado, C. J., \& Platnick, N. I. (2008). Review of the southern South American spider genus Platnickia (Araneae, Zodariidae). American Museum Novitates, 3625, 1-19.

Guzmán, J. A., D’Elía, G., \& Ortíz, J. C. (2009). Variación geográfica del zorro Lycalopex culpaeus (Mammalia, Canidae) en Chile: implicaciones taxonómicas. Revista de Biología Tropical, 57, 421-432.

Hünicken, M. A., Charrier, R., \& Lahsen, A. (1980). Baculites (Lytoceratina) de la base de la Formación Fuentes (Campaniano Medio - Superior) de la Isla Riesco, Provincia de Magallanes, Chile. Boletín de la Academia Nacional de Ciencias, Córdoba, 53, 221235.

Ibar, E. (1878). Hidrografía: Estudios sobre las aguas de Skyring i la parte austral de la Patagonia por el comandante y oficiales de la corbeta Magallanes: Memoria de don Enrique Ibar Sierra. Anales de la Universidad de Chile, noviembre 1878, 579-637.

Imberti, S. (2005). Distribución otoñal de aves marinas y terrestres en los canales chilenos. Anales del Instituto de la Patagonia, 33, 21-30.

Jaksic, F. M. (2001). Spatiotemporal variation patterns of plants and animals in San Carlos de Apoquindo, central Chile. Revista Chilena de Historia Natural, 74, 477-502. Jaramillo, A., Burke, P., \& Beadle, D. (2003) Birds of Chile. London, UK: Christopher Helm.

Jiménez, J. E. (2010). Southern pudu Pudu puda (Molina, 1782). En J. M. Barbanti \& S. González (Eds.), Neotropical Cervidology: Biology and Medicine of Latin American Deer (pp. 140-150). Jaboticabal \& Gland: Funep \& IUCN.

Kusch, A., \& Marín, M. (2010). Distribución de la becasina grande Gallinago stricklandii (Gray, 1845) (Scolopacidae), en Chile. Anales del Instituto de la Patagonia, 38, 145-149.
Kusch, A., Ramírez, I., Vila, A., Droguett, D., Paulsen, K., Cabello, J., ..., \& Chauveau, M. (2016). Resultados del Taller "Actualización del Plan de Acción para la conservación del huemul Hippocamelus bisulcus (Molina, 1782) en la zona austral de Chile". Anales del Instituto de la Patagonia , 44, 61-72.

Latorre, J. J. (1879). Esploración de las Aguas de Skyring o del Despejo i de la parte austral de la Patagonia. Anuario Hidrográfico de la Marina de Chile, 5, 3-46.

Lefèvre, C., Lepetz, S., \& Legoupil, D. (2003). ¿Cazadores terrestres, cazadores marítimos? Explotación de los recursos animales en el Locus 1. Magallania, 31, 63-116.

Legoupil, D. (2003). Cazadores-recolectores de Ponsonby (Patagonia Austral) y su paleoambiente desde VI al III milenio AC. Magallania, 31 (Volumen especial).

Lepetz, S., Lefèvre, C., \& Pellé, E. (2003). Los guanacos de la turbera: nota sobre un depósito natural. Magallania, 31, 415-418.

Leppe, M., Mihoc, M., Varela, N., Stinnesbeck, W., Mansilla, H., Bierma, H.,... Jujihara, T. (2012). Evolution of the Austral-Antarctic flora during the Cretaceous: New insights from a paleobiogeographic perspective. Revista Chilena de Historia Natural, 85, 369-392.

Mann, G. (1978). Los pequeños mamíferos de Chile. Gayana, zoología, 40, 1-342.

Markham, B.J. (1970). Reconocimiento faunístico del área de los fiordos Toro y Cóndor, Isla Riesco, Magallanes. Anales del Instituto de la Patagonia, 1, 41-59.

Markham, B.J. (1971a). Catálogo de los anfibios, reptiles, aves y mamíferos de la provincia de Magallanes (Chile). Publicaciones del Instituto de la Patagonia, serie Monografía, 1, 1-64.

Markham, B.J. (1971b). Notas sobre las subespecies del "culpeo" (Dusicyon culpaeus) en Magallanes. Anales del Instituto de la Patagonia, 2, 166-169.

Markham, B.J. (1971c). Descripción de una nueva subespecie de "tordo" Curaeus curaeus recurvirostris, subsp. nov. Anales del Instituto de la Patagonia, 2, 158-159.

Martinic, M. (1957). Fauna magallánica, IV. Los 
roedores (Macro-roedores) y los Quirópteros. Boletín Ganadero, XX, 20-21.

Martinic, M. (2005). Documentos para la historia de Magallanes. Recuerdos e impresiones de dos inmigrantes alemanes en Magallanes. Magallania, 33, 121-146.

Martinic, M. (2011). A la hora del crepúsculo: recuerdos de un hombre común. Punta Arenas, Chile: Universidad de Magallanes.

Matus, R., \& Jaramillo, A. (2008). Range extensions and vagrant bird species in the XII Region of Magallanes, Chile. Cotinga, 30, 34-40.

Miller, S. D., Rottmann, J., Raedeke, K. J., \& Taber, R. D. (1983). Endangered mammals of Chile: status and conservation. Biological Conservation, 25, 335-352.

MMA (Ministerio del Medio Ambiente) (2013). Aprueba y oficializa Clasificación de Especies según su Estado de Conservación, Noveno Proceso. Decreto Supremo 13/2013. Disponible en https://www. leychile.cl/Navegar?idNorma $=1053125$.

Molina, R., Soto, N., \& Tapia, A. (2018). Estado actual de la distribución del castor Castor canadensis Kuhl 1820 (Rodentia) en el área continental de la región de Magallanes, Chile. Anales del Instituto de la Patagonia, 46, 7-15.

Moreira-Arce, D., Ugarte, C. S., ZorondoRodríguez, F., \& Simonetti, J. A. (2018). Management tools to reduce carnivorelivestock conflicts: current gap and future challenges. Rangeland Ecology \& Management, 71, 389-394.

Ojeda, M. E., \& Pérez, H. S. (2009). La caza del puma en Magallanes. Una mirada histórica-actual del conflicto entre el depredador y la actividad ganadera. Tesis de Profesor de Historia y Ciencias Sociales. Punta Arenas, Chile: Facultad de Humanidades y Ciencias Sociales y de la Salud, Universidad de Magallanes.

Ortíz, J. C. (2015). Anfibios de las turberas del extremo austral de Chile. Cap. 8 pp. 229 - 240. En E. Domínguez \& D. Vega-Valdés (Eds.), Funciones y servicios ecosistémicos de las turberas en Magallanes. Punta Arenas, Chile: Instituto de Investigaciones
Agropecuarias, Centro Regional de Investigación Kampenaike.

Osgood, W. H. (1943). The mammals of Chile. Field Museum of Natural History, Zoological Series, 30, 1-168.

Palma, R. E., \& Rodríguez-Serrano, E. (2018). Systematics of Oligoryzomys (Rodentia, Cricetidae, Sigmodontinae) from southern Chilean Patagonia, with the description of a new species. Journal of Zoological Systematics and Evolutionary Research, 56, 280-299.

Palma, R. E., Boric-Bargetto, D., Torres-Pérez, F., Hernández, C. E., \& Yates, T. L. (2012). Glaciation effects on the phylogeographic structure of Oligoryzomys longicaudatus (Rodentia: Sigmodontinae) in the Southern Andes. PLoS ONE, 7, e32206.

Pérez, V. (2018). Paleontología de la Región de Magallanes: Guía bibliográfica 1846-2010. Parte II. Vertebrados. Anales del Instituto de la Patagonia, 44, 51-77.

Pérez, E., \& Pérez, V. (2018). Paleontología de la Región de Magallanes: Guía bibliográfica 1846-2010. Parte I. Invertebrados. Anales del Instituto de la Patagonia, 43, 75-89.

Pisano, E. (1977). Contribución de Enrique Ibar Sierra al conocimiento de la naturaleza de Patagonia Occidental Austral. Anales del Instituto de la Patagonia, 8, 95-119.

Povilitis, A., \& G. Hughes(Eds.) (2003). Prospección para huemules en la Isla Riesco, Sector Río León, XII Región, Chile. California, EE.UU.: Sierra Institute, Programa de Extensión Universidad de California-Santa Cruz.

Ramírez, M. J. (2003). The spider subfamily Amaurobioidinae (Araneae, Anyphaenidae): a phylogenetic revision at the generic level. Bulletin of the American Museum of Natural History, 277, 1-262.

Rodríguez-Serrano, E.A. (2011). Consecuencias de las glaciaciones Pleistocénicas sobre la diversidad genética de Abrothrix olivaceus (Waterhouse) (Rodentia: Cricetidae: Sigmodontinae). Tesis Doctoral. Santiago, Chile: Facultad de Ciencias Biológicas, Pontificia Universidad Católica de Chile.

Saavedra, C. (2018). Uso de repelentes olfativos como alternativa de control no letal para 
el manejo de carnívoros silvestres. Tesis de Magister. Santiago, Chile: Facultad de Ciencias, Universidad de Chile.

Salgado, J. M. (2005). Description of new genera and species of the subtribe Eunemadina from Chile. New data on other genera (Coleoptera, Leiodidae, Cholevinae). Deutsche Entomologische Zeitschrift, 52, 191-216.

Salgado, J. M. (2006). Nuevas aportaciones al conocimiento de Camiarinae y Cholevinae (Coleoptera: Leiodidae) Neotropicales y descripción de una nueva especie de Chile. Boletín del Museo Nacional de Historia Natural, Chile, 55, 87-111.

San Román, M., Morello, F., \& Prieto, A. (2002). Nuevos antecedentes sobre la explotación de recursos faunísticos en el mar de Otway y canales adyacentes. Anales del Instituto de la Patagonia, 30, 147-154.

Sielfeld, W. (1979). Presencia de Microcavia australis (G y $\left.D^{\prime} \mathrm{O}\right)$ en Magallanes (Mammalia: Caviidae). Anales del Instituto de la Patagonia, 10, 197-199.

Sielfeld, W. (1997). Las áreas protegidas de la XII Región de Chile en la perspectiva de los mamíferos marinos. Estudios Oceanológicos, 16, 87-107.

Silva, M. (2002). Día Quinto. Santiago, Chile: Editorial Universitaria.

Silva, C., Repetto, F., Droguett, D., Moraga, C., \& Vila, A. (2011). Actas de Taller: Hacia un plan para la conservación del Huemul Hippocamelus bisulcus (Molina, 1782) en la zona austral de Chile. Anales del Instituto de la Patagonia, 39, 119-136.

Simonetti, J. A., \& Stipicic, G. J. (2016). Primer registro de Rara (Phytotoma rara Molina, 1782) en Isla Riesco, Magallanes. Anales del Instituto de la Patagonia, 44, 77-79.

Simonetti, J. A., Stipicic, G. J., SimonettiGrez, G., \& Grez, A. A. (2016). Bombus terrestris (Linnaeus) silvestres en Isla Riesco, Magallanes (Hymenoptera: Apidae). Revista Chilena de Entomología, 41, 91-94.

Snelling, R. R., \& Hunt, J. H. (1975). The ants of Chile (Hymenoptera: Formicidae). Revista Chilena de Entomología, 9, 63-129.
Solari, M. E., Bernard, V., Legoupil, D., Richard, H., Roiron, P., \& Schoellammer, P. (2002). Palaeoenvironmental approach of Ponsonby archaeological site (Riesco Island, Chilean Patagonia). En S. Thiébault (Ed.), Charcoal analysis. Methodological approaches, palaeoecological results and wood uses. British Archaeological Reports, International Series, 1063, 179-185.

Stipicic, G. J., Simonetti-Grez, G., \& Simonetti, J. A. (2017). Primer registro de canquén colorado (Chloephaga rubidiceps, Sclater, 1861) (Aves: Anatidae) en Isla Riesco, Magallanes. Anales del Instituto de la Patagonia, 45, 117-119.

Tamayo, M., \& Frassinetti, D. (1980). Catálogo de los mamíferos fósiles y vivientes de Chile. Boletín del Museo Nacional de Historia Natural, Chile, 37, 323-399.

Taucare-Ríos, A. (2014). Mecysmauchenius segmentatus (Araneae: Mecysmaucheniidae) en Isla Riesco: ampliación de su distribución conocida en Magallanes, Chile. Anales del Instituto de la Patagonia, 42, 77-79.

Venegas, C. \& Sielfeld, W. (1998). Catálogo de los vertebrados de la Región de Magallanes y Antártica Chilena. Punta Arenas, Chile: Ediciones de la Universidad de Magallanes.

Wheelwright, N. T. (1978). Snowy egret in the Strait of Magellan. Auk, 95, 590.

Zorondo-Rodríguez, F., Díaz, M., SimonettiGrez, G., \& Simonetti, J. A. (2019). Why would new protected areas be accepted or rejected by people? Lessons from an exante evaluation of the new Patagonia Park Network in Chile. Land Use Policy, 89, 104248.

Zúñiga-Reinoso, A. (2011). Los coccinélidos (Coleoptera: Coccinellidae) de la Región de Magallanes: nuevos registros y distribución regional. Anales del Instituto de la Patagonia, 39, 59-71.

Zúñiga-Reinoso, A. (2013). Revisión de los Cerambycidae (Coleoptera) de la Región de Magallanes: lista ilustrada. Anales del Instituto de la Patagonia, 41, 53-59. 